\title{
Stationary-Phase Complexation of Crown Ethers with Polyammonium Ions Chemically Bonded on Polymer Resin
}

\author{
Sujata S. BISWAS and Tetsuo OKADA ${ }^{\dagger}$ \\ Department of Chemistry, Tokyo Institute of Technology, Meguro, Tokyo 152-8551, Japan
}

\begin{abstract}
The apparent comlexation of crown ethers with polyammonium ions chemically bonded on polystyrene-divinylbenzene copolymer resin has been chromatographically studied in methanol and acetonitrile. The retention of crown ethers with chemically bonded ammonium ion resins is affected by the nature of the counteranions; the retention is enhanced with large and poorly solvated counteranions, but is reduced with small and well solvated counteranions. In the present study, the retention mechanisms are discussed based on the retention data obtained by changing various factors, such as the crown cavity sizes, the structures of the polyammonium ions, counteranions, and the molar ratios of mixed counteranions in the stationary phase. The complexation and ion-pair formation equilibria play important roles in determining the overall retention of crown ethers.
\end{abstract}

Keywords Crown ethers, polyammonium ions, complexation, stationary phases

Crown ether complexation has received much attention, though more than three decades have passed since this class of compounds was first synthesized by Pedersen. ${ }^{1}$ One typical question in crown-ether chemistry is how the selectivity in crown ether complexation emerges. Understanding the origin of the selectivity in crown-ether complexation is not only of fundamental interest, but also of practical importance. Various thermodynamic ${ }^{2-4}$, structural ${ }^{5-8}$, and computational research efforts ${ }^{9,10}$ have been conducted to answer this question. The size-fit theory was a widely accepted concept to explain crown-ether complexation selectivity, and is still regarded as a dominant factor in some instances. However, there are a number of examples in which the selectivity does not follow simple size-fit theories. ${ }^{11}$ Thus, it is reasonable to regard size-fit as one of the important factors governing crown ether complexation selectivity.

Crown ether complexation with simple metal cations has been most extensively studied, because simple inorganic cations are well characterized; thus, a number of reliable physicochemical data are available, which allow us to quantitatively discuss the thermodynamic and structural aspects of crown ether complexes. This background in fundamental chemistry has allowed us to develop a variety of analytical methods based on crown ether complexation. Crown ethers have been utilized for separation ${ }^{12-14}$, ion selective electrodes ${ }^{15,16}$, and optical sensors ${ }^{17,18}$ for simple inorganic cations (mainly metal cations). In contrast, studies on the complexation

S. S. B. permanent address: Division of Organic Chemistry, National Chemical Laboratory, Pune 411 008, India.

† To whom correspondence should be addressed. of crown ethers with organic cations have been relatively few. However, this does not necessarily mean that organic cations are less important than inorganic cations in crown ether chemistry; on the contrary, complexation with organic cations including the conjugate acids of amines and aminoacids is expected to become more important in biological, biochemical, medical, and pharmaceutical sciences.

For investigating polyether complexation, we have developed efficient means based on separation techniques, such as cation-exchange chromatography ${ }^{19-22}$, anion-exchange chromatography ${ }^{23,24}$, and capillary electrophoresis. ${ }^{25}$ These methods have permitted us to elucidate polyether chemistry, which is difficult to study with conventional spectrometric, electrochemical, and thermodynamic methods. In the present work, we chromatographically studied the complexation behaviors of crown ethers with polyammonium ions anchored on a polymer resin. The purposes of this work were (1) to characterize the retention of crown ethers in these stationary phases, (2) to elucidate the phenomena which take place in or on the stationary phases, and (3) to show that the stationary phases are useful to study polyether complexation. The complexation of crown ethers with polyammonium ions has not been investigated very well, even in solution. Thus, the present method is expected to elucidate important aspects of crown ether complexation with polyammonium ions.

\section{Experimental}

The chromatographic system was composed of a 


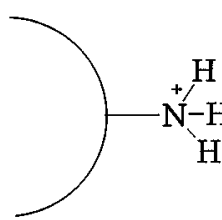

$-\mathrm{NH}_{3}^{+}$

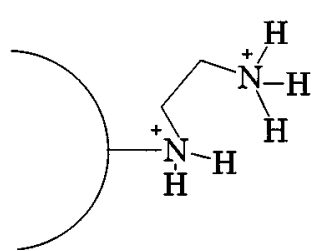

$-\mathrm{en}^{2+}$

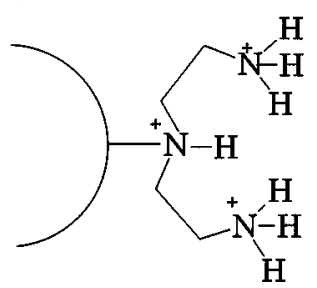

- dien $^{3+}$

Fig. 1 Structures of the active sites of polyammonium resins.

Tosoh computer-controlled pump Model CCPD, a Reodyne injection valve equipped with a $100 \mu \mathrm{l}$ sample loop, a JASCO UV-visible detector Model 875-UV, and a chart recorder. The separation column was immersed in water thermostated at $25.0^{\circ} \mathrm{C}$. Stationary phases bearing ammonium ions were synthesized from MCI GEL 5HP (polystylene-polydivinylbenzene copolymer, Mitsubishi Chemicals, the mean particle size is $5 \mu \mathrm{m}$ ) by chloromethylation, followed by a reaction with an appropriate amine: (1) ammonia $\left(-\mathrm{NH}_{3}{ }^{+}\right)$, (2) ethylendiamine $\left(-\mathrm{en}^{2+}\right)$, and (3) diethylenetriamine $\left(-\mathrm{dien}^{3+}\right)$. The schematic representation of the structures of active groups is depicted in Fig. 1. To avoid any structural variation in the dien ${ }^{3+}$ stationary phase, diethylenetriamine was allowed to react with the chloromethylated resin after protecting both terminal primary amino groups by a reaction with salicylaldehyde. Thus, dien is anchored on the resin at the middle nitrogen, as shown in Fig. 1. The stationary phases were packed in a PTFE column $(4.6 \mathrm{~mm}$ i.d. $\times 50 \mathrm{~mm})$, and then completely protonated by passing an appropriate acid solution. Crown ethers having different cavity sizes, i.e. benzo-15-crown-5 (B15C5), dibenzo-18-crown-6 (DB18C6), dibenzo-24-crown-8 (DB24C8), and dibenzo-30-crown-10 (DB30C10), were synthesized according to the literature. ${ }^{1}$ The reagents and solvents were of analytical grade.

The amount of amines in the stationary phase was determined as follows: a stationary phase was equilibrated with a $\mathrm{NO}_{3}{ }^{-}$solution; after rinsing the column with water, passing a sufficient volume of a $\mathrm{NaClO}_{4}$ solution allowed the complete replacement of $\mathrm{NO}_{3}{ }^{-}$by UV-transparent $\mathrm{ClO}_{4}^{-}$; the concentration of $\mathrm{NO}_{3}{ }^{-}$in effluents was then spectrophotometrically determined.

The complex formation constants $(K)$ of crown ethers in $\mathrm{MeOH}$ were determined chromatographically with the $-\mathrm{NH}_{3}{ }^{+} \mathrm{ClO}_{4}{ }^{-}$resins. When the mobile phases con- tain a complex-forming cation ( $C$ is its concentration), the retention factor of the crown ethers decreases with increasing concentration of the salt according to

$$
k^{\prime}=\frac{k_{0}{ }^{\prime}}{1+K C},
$$

where $k_{0}{ }^{\prime}$ is the retention factor obtained with a mobile phase not containing complex forming cations. The complexation constants were thus determined from the dependence of the retention on the concentration of the salt added in the mobile phases. $\mathrm{ClO}_{4}^{-}$salts were used as mobile-phase additives to avoid ion-pair formation of the added salts. Perchlorate salts of polyammonium ions were synthesized by mixing the polyamine with equivalent aqueous perchloric acid followed by recrystallization from an appropriate solvent.

\section{Results and Discussion}

\section{Retention of crown ethers on ammonium resins}

Crown ethers are not retained on the stationary phases when the amine groups on the stationary phase surface are not protonated. In addition, the nature of the counteranions is well reflected on the retention of crown ethers. These facts suggest that the crown ethers are retained on the resins used in this work by their complexation with ammonium groups.

It is significant to compare the crown ether affinity to different active groups (varying both polyammonium ions and counteranions). However, the retention times (or capacity factors) of crown ethers do not necessarily reflect their affinity to the stationary phase, because the surface density of the active groups is not identical. The following retention index $(I)$ was therefore used to compare the retention ability of the stationary phases:

$$
I=k^{\prime} / m_{\mathrm{am}}
$$

where $m_{\mathrm{am}}$ is the total mole of active molecules anchored on the stationary phase; $m_{\mathrm{am}}=6.73 \times 10^{-5} \mathrm{~mol}$ for the $-\mathrm{NH}_{3}{ }^{+}, m_{\mathrm{am}}=1.33 \times 10^{-4} \mathrm{~mol}$ for the $-\mathrm{en}^{2+}$, and $m_{\mathrm{am}}=7.31 \times 10^{-5} \mathrm{~mol}$ for the $-\mathrm{dien}^{3+}$ resin per column. This index $(I)$ is just a parameter representing the approximate retention (or complexation) ability per 1 mol of active groups, but has no rigorous physical meaning. The indices are listed in Table 1.

Crown ether complexation with ammonium ions chemically bonded on solid phases can be roughly explained as follows (as shown in Fig. 2): (1) the dissociation of an ion-pair between the ammonium ion and a counteranion; (2) the complexation of a crown ether with the ammonium ion; and (3) ion-pair formation between an counteranion and the ammonium ion complexed by a crown ether. Thus, the nature of an anion is reflected in equilibria (1) and (3), while the properties of a crown ether are involved in equilibria (2) and (3). Equilibrium (1) is common to all crown ethers for 
Table 1 Retention indices defined by Eq. (1)

\begin{tabular}{|c|c|c|c|c|c|c|c|c|c|c|c|c|}
\hline \multirow{3}{*}{$\begin{array}{l}\text { Crown } \\
\text { ether }\end{array}$} & \multicolumn{6}{|c|}{$I\left(\mathrm{ClO}_{4^{-}}^{-}\right) / 10^{5}$} & \multicolumn{6}{|c|}{$I\left(\mathrm{NO}_{3}^{-}\right) / 10^{5}$} \\
\hline & \multicolumn{2}{|c|}{$-\mathrm{NH}_{3}{ }^{+}$} & \multicolumn{2}{|c|}{$-\mathrm{en}^{2+}$} & \multicolumn{2}{|c|}{$-\operatorname{dien}^{3+}$} & \multicolumn{2}{|c|}{$-\mathrm{NH}_{3}{ }^{+}$} & \multicolumn{2}{|c|}{$-\mathrm{en}^{2+}$} & \multicolumn{2}{|c|}{$-\operatorname{dien}^{3+}$} \\
\hline & $\mathrm{AN}$ & $\mathrm{MeOH}$ & $\mathrm{AN}$ & $\mathrm{MeOH}$ & AN & $\mathrm{MeOH}$ & AN & $\mathrm{MeOH}$ & $\mathrm{AN}$ & $\mathrm{MeOH}$ & AN & $\mathrm{MeOH}$ \\
\hline B15 & 1.4 & 0.55 & 0.75 & 0.47 & 1.2 & 0.82 & 0.55 & 0.31 & 0.23 & 0.11 & 0.45 & 0.22 \\
\hline DB18C6 & - & - & 1.7 & - & 2.6 & - & 2.4 & 2.3 & 0.36 & 0.72 & 0.59 & 1.8 \\
\hline DB24C8 & 1.1 & 0.75 & 0.60 & 0.72 & 0.74 & 1.3 & 0.34 & 0.34 & 0.094 & 0.11 & 0.26 & 0.22 \\
\hline DB30C10 & 0.68 & 0.55 & 1.0 & 1.0 & 0.52 & 0.89 & 0.21 & 0.27 & 0.075 & 0.075 & 0.19 & 0.15 \\
\hline
\end{tabular}
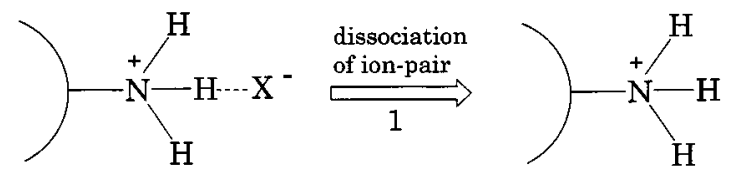

retention
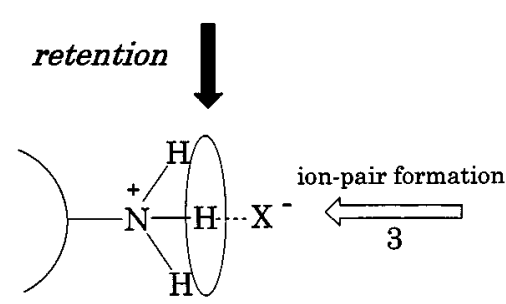

$2]$ complexation

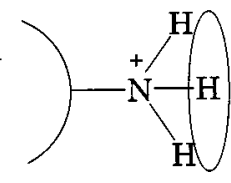

Fig. 2 Schematic representation of the retention mechanisms.

a particular counteranion. When the contribution from equilibrium (3) is much smaller than equilibrium (2), the retention selectivity should simply reflect the stationary phase complexation. In contrast, if the retention does not follow simple complexation mechanisms, equilibrium (3) plays a major part in determining the overall retention. Thus, a comparison of the retention of crown ethers with their complexation in solutions gives insights into the mechanism of the resin phase complexation.

Roles of complexation equilibria in the overall retention

It is advantageous to first discuss the retention selectivity with $\mathrm{ClO}_{4}{ }^{-}$resins in $\mathrm{MeOH}$, because $\mathrm{ClO}_{4}{ }^{-}$sites are almost completely dissociated and retention is expected to be dominantly governed by the complexation mechanism. A capillary electrophoresis study indicated that alkylammonium ions are similarly complexed by B15C5, DB24C8, and DB30C10 in methanol. ${ }^{25}$ If the complexation of crown ethers with the anchored ammonium ions is replaced by that with the alkylammonium ion in solution, these crown ethers should show similar retention. In fact, there are very small differences in the indices $(I)$ for the $-\mathrm{NH}_{3}{ }^{+}$resin between these crown ethers. This supports the inference that the complexation equilibrium is dominantly responsible for the retention process. The retention indices for DB18C6 cannot be determined, because it was not eluted within reasonable analysis times. The complexation constants of DB18C6 in $\mathrm{MeOH}$ are much larger than those of other crown ethers tested by at least
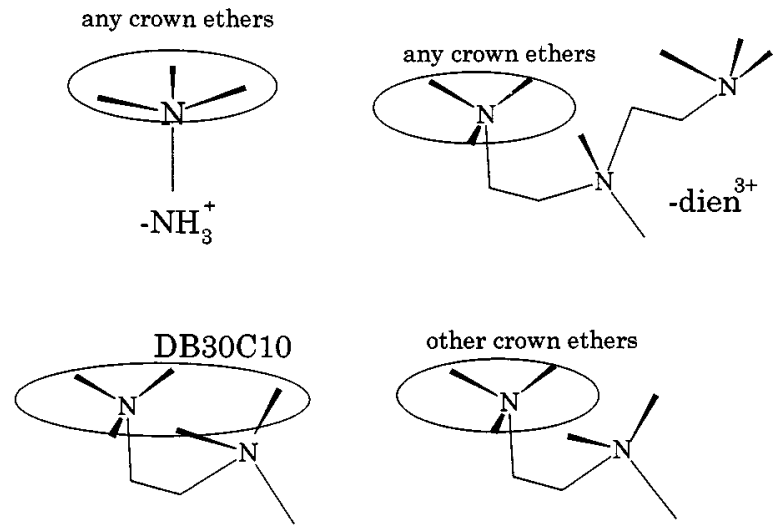

$-\mathrm{en}^{2+}$

Fig. 3 Possible structures of crown ether complexes with polyammonium ions anchored on the polymer resin.

one order of magnitude; ${ }^{11}$ this also agrees with a chromatographic observation. Interestingly, the retention properties of the - dien $^{3+}$ resin are similar to those of the $-\mathrm{NH}_{3}{ }^{+}$resin; DB24C8 complexation ability is slightly larger than those of $\mathrm{B} 15 \mathrm{C} 5$ and $\mathrm{DB} 30 \mathrm{C} 10$. This indicates that terminal primary ammonium groups of the dien $^{3+}$ molecule individually interact with crown ethers.

Although the retention ability of DB30C10 is rather weak in most cases, specifically high retention occurs with the en ${ }^{2+}$ resin. This implies a specific interaction between DB30C10 and the $-\mathrm{en}^{2+}$ sites. The large ring size of DB30C10 possibly allows the simultaneous inclusion of primary and secondary ammonium ions in an $\mathrm{en}^{2+}$ molecule. Our previous capillary electrophoresis study indicated that a secondary ammonium ion (e.g. diethylammonium ion) forms complexes with crown ethers in $\mathrm{MeOH}$ albeit the interaction is weaker than that with primary ammonium ions. ${ }^{25}$ A possible structure of DB30C10-en ${ }^{2+}$ is schematically illustrated in Fig. 3. If the en ${ }^{2+}$ molecule adopts a gauche conformer along its $\mathrm{C}-\mathrm{C}$ bond, the distance between the terminal ammonium ions is estimated to be $<3 \AA$. Though the accurate ring size of DB30C10 in solution is unknown, the diameter of the cavity should be greater than $3 \AA$. DB30C 10 can thus accommodate two ammonium ions in an $\mathrm{en}^{2+}$ molecule in its cavity. DB30C10 basically forms a complex with the primary ammonium ion in an $e^{2+}$ molecule, and additionally interacts with the secondary ammonium ion in the same 
Table 2 Complex formation constants of crown ethers with ammonium ions

\begin{tabular}{lccc}
\hline \multirow{2}{*}{$\begin{array}{c}\text { Crown } \\
\text { ether }\end{array}$} & \multicolumn{3}{c}{$\log K$} \\
\cline { 2 - 4 } & $\mathrm{PrNH}_{3}{ }^{+}$ & $\mathrm{en}^{2+}$ & en-EtOH \\
& & \\
\hline B15C5 & $1.44 \pm 0.06$ & $2.04 \pm 0.06$ & $1.65 \pm 0.03$ \\
DB18C6 & $2.23 \pm 0.02$ & $2.56 \pm 0.15$ & $2.23 \pm 0.03$ \\
DB30C10 & $1.43 \pm 0.06$ & $2.77 \pm 0.03$ & $2.14 \pm 0.03$ \\
\hline
\end{tabular}

$\mathrm{en}^{2+}$ molecule to enhance the stability of the complex. This phenomena cannot be observed for the dien ${ }^{3+}$ resin, because the interaction with the tertiary ammonium ion is much weaker and the distance between two primary ammonium ions in this molecule (longer than $5.3 \AA$, depending on the conformation of this molecule) is too long even for DB30C10 to accommodate two primary ammonium ions simultaneously in its cavity. Thus, it can be reasonably concluded that the structure of a DB30C10-dien ${ }^{3+}$ complex is similar to that of a DB30C10- $\mathrm{NH}_{3}{ }^{+}$complex, but different from that of a DB30C10-en ${ }^{2+}$ complex (see Fig. 3 ).

To confirm the above consideration, the $1: 1$ complexation constants $(K)$ of crown ethers with $\mathrm{PrNH}_{3}{ }^{+}, \mathrm{N}-2$ hydroxyethyl-ethylenediammonium (en-EtOH ${ }^{2+}$ ), and $\mathrm{en}^{2+}$ in $\mathrm{MeOH}$ were chromatographically determined, as summarized in Table 2. The differences in the $\log K$ values between $\mathrm{PrNH}_{3}{ }^{+}$and en-EtOH ${ }^{2+}$ are $0.21 \pm 0.07$ for $\mathrm{B} 15 \mathrm{C} 5,0 \pm 0.04$ for $\mathrm{DB} 18 \mathrm{C} 6$, and $0.71 \pm 0.07$ for DB30C10, while those between en-EtOH ${ }^{2+}$ and en ${ }^{2+}$ are $0.39 \pm 0.07$ for $\mathrm{B} 15 \mathrm{C} 5,0.33 \pm 0.15$ for DB $18 \mathrm{C} 6$, and $0.63 \pm 0.04$ for DB30C10, respectively. For B $15 \mathrm{C} 5$ and DB18C6, the $\Delta \log K$ between en-EtOH ${ }^{2+}$ and $\mathrm{en}^{2+}$ can be explained by a statistic effect due to increasing number of primary ammonium groups; increasing the number of ammonium groups, from one to two, corresponds to a 0.30 increase in $\log K$. The $\log K$ values for DB30C10-diammonium ions are obviously larger than expected from that with the monoammonium ion, suggesting that structural stabilization occurs in the DB30C10 complexes with the diammonium ions. Thus, the specific interaction between DB30C10 and $\mathrm{en}^{2+}$ has been chromatographically discovered with the $\mathrm{en}^{2+}$ resins.

There are a few data for crown ether complexation in AN. Although we attempted to determine the crown ether complexation constants in solutions, it was difficult to obtain reliable values because of the association of ions in AN. This makes it difficult to discuss the retention of crown ethers based on their solution phase complexation. However, since ion-pair formation equilibria do not contribute to the retention on the $-\mathrm{NH}_{3}{ }^{+} \mathrm{ClO}_{4}{ }^{-}$resin (see below), the retention indices obtained in this system should well reflect the contribution from the complexation process. The retention of any crown ethers in $\mathrm{AN}$ is larger than in $\mathrm{MeOH}$, indicating stronger complexation in AN. As mentioned below, the ion-association between the anchored ammonium ions and counteranions is enhanced as the charges of the interacting ions increase. This may result in smaller retention indices in $\mathrm{AN}$ for some crown ethers. However, all of the results cannot be explained (e.g. B15C5 always shows larger retention indices in AN) because solvent effects on the crown ether complexation are very complex. ${ }^{26,27}$

\section{Ion-pair formation of polyammonium ions with counter- anions}

The ion-pair formation behaviors of appropriate compounds in solution allow one to infer stationary phase ion-pair formation behaviors of polyammonium ions on the stationary phase. The conductivity of propylammonium $\left(\mathrm{PrNH}_{3}{ }^{+}\right)$, ethylenediammonium $\left(\mathrm{en}^{2+}\right)$, and diethylenetriammonium $\left(\mathrm{dien}^{3+}\right) \mathrm{NO}_{3}{ }^{-}$and $\mathrm{ClO}_{4}{ }^{-}$was measured in $\mathrm{MeOH}$ and $\mathrm{AN}$. The results are illustrated in Fig. $4\left(\Lambda / \Lambda^{\infty}-C\right.$, the ratios of molar conductivity to the limiting molar conductivity are plotted $v s$. the concentrations of salts). If no ion-pairs are formed, the concentration dependence of $\Lambda$ should follow e.g. Onsager equations:

$$
\begin{aligned}
& \lambda=\left(\lambda^{\circ}-\left(\frac{\left|z_{+}+\right| z_{-} \mid e^{2}}{12 \pi \varepsilon_{0} \varepsilon k T} \frac{q}{1+\sqrt{q}} \lambda^{\circ}+\frac{F^{2}|z|}{6 \pi \eta N}\right)\right) \frac{\kappa}{1+\kappa a} \\
& q=\frac{\left|z_{+} z_{-}\right|}{\left|z_{+}+\right| z_{-} \mid} \frac{\lambda_{+}^{\circ}+\lambda_{-}^{\circ}}{\left|z_{+}\right| \lambda_{-}^{\circ}+z_{-}-\lambda_{+}^{\circ}},
\end{aligned}
$$

where $\varepsilon_{0}, \varepsilon, \eta, \kappa, \lambda^{0}, e, F, k, N$, and $T$ are the permittivity of the vacuum, the relative permittivity of the medium, the viscosity of the medium, the Debye screening parameter, the limiting molar ionic conductivity, the elementary charge, the Faraday constant, the Boltzmann constant, the Avogadro's number, and the temperature, respectively; subscripts "+" and "-" represent a cation and an anion.

The relative molar conductivity calculated with an Onsager equation is also shown in Fig. 4. $\mathrm{PrNH}_{3} \mathrm{ClO}_{4}$ almost follows this equation both in $\mathrm{MeOH}$ and in $\mathrm{AN}$. However, in other cases, $\Lambda$ decreases more steeply than expected from this theory. Although the molar conductivity of some salts first increases, and then decreases with increasing concentration due to the dissociation of polyammonium ions in $\mathrm{MeOH}$, the slopes of the decreasing parts in the $\Lambda / \Lambda^{\infty}-C$ plots are much larger than from the corresponding calculations. A comparison of the decreasing parts in the $\Lambda / \Lambda^{\infty}-C$ curves gives approximate trends of the ion-pair formation of these salts. Ion-pair formation is enhanced in the order of $\mathrm{PrNH}_{3}{ }^{+}<\mathrm{en}^{2+}<\mathrm{dien}^{3+}, \mathrm{MeOH}<\mathrm{AN}$, or $\mathrm{ClO}_{4}{ }^{-}<\mathrm{NO}_{3}{ }^{-}$. Conductometric measurements for the $\mathrm{en}^{2+}$ and $\mathrm{dien}^{3+}$ $\mathrm{NO}_{3}{ }^{-}$salts in AN were not possible because they are not soluble in this solvent. However, their low solubility implies stronger bindings between $\mathrm{NO}_{3}{ }^{-}$and polyammonium cations in AN. For dien ${ }^{3+}$ in AN, ion-pair formation is not negligible even for the $\mathrm{ClO}_{4}{ }^{-}$counteranion. 

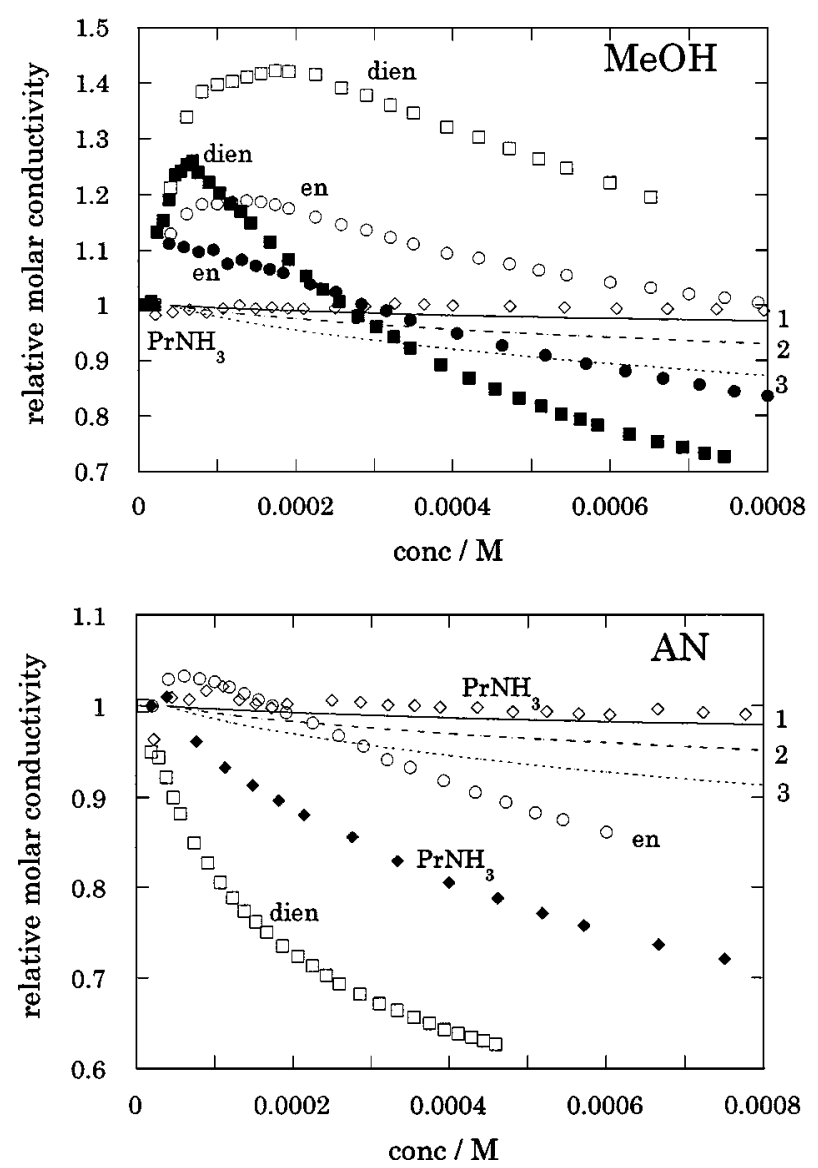

Fig. $4 \Lambda / \Lambda^{\infty}-C$ plots for ammonium salts in $\mathrm{MeOH}$ and $\mathrm{AN}$. Open symbols, $\mathrm{ClO}_{4}^{-}$salts. Solid symbols, $\mathrm{NO}_{3}{ }^{-}$salts. Curves 1 - 3 show Onsager's prediction of $\Lambda / \Lambda^{\infty}$ for $1: 1,1: 2$, and $1: 3$ salts, respectivery.

\section{Role of ion-pair formation}

Our previous nonaqueous ion-exchange chromatographic experiments ${ }^{28}$ indicated that the affinity of $\mathrm{ClO}_{4}^{-}$to anion-exchange resins (both primary and tertiary ammonium ion types) is much weaker than $\mathrm{NO}_{3}$ in $\mathrm{AN}$; for example, we cannot measure the retention times of $\mathrm{ClO}_{4}^{-}$with $\mathrm{NO}_{3}{ }^{-}$as an eluent because of the extremely weak retention ability of the former, while $\mathrm{NO}_{3}{ }^{-}$is not eluted with $\mathrm{ClO}_{4}{ }^{-}$as an eluent. This clearly implies that $\mathrm{NO}_{3}{ }^{-}$is very tightly bound by anionexchange sites compared to $\mathrm{ClO}_{4}^{-}$. This enhanced ionpair formation makes the crown ether retention weaker through equilibrium (1). Although the anion-exchange chromatographic results indicate very large differences in the affinity to the resin between these anions (possibly $2-3$ orders of magnitude), the retention indices of the crown ethers on the $\mathrm{NO}_{3}{ }^{-}$-form resins in $\mathrm{AN}$ remain half to one-third as large as that on the $\mathrm{ClO}_{4}^{-}$-form resins; if equilibrium (1) dominantly determines the total retention, there should be much larger differences in the retention between these two counteranions. This implies that the effects of equilibrium (1) are almost compensated by equilibrium (3). If the ion-pair formation constants $\left(K_{1}\right.$ and $\left.K_{3}\right)$ are defined for equilibria (1)
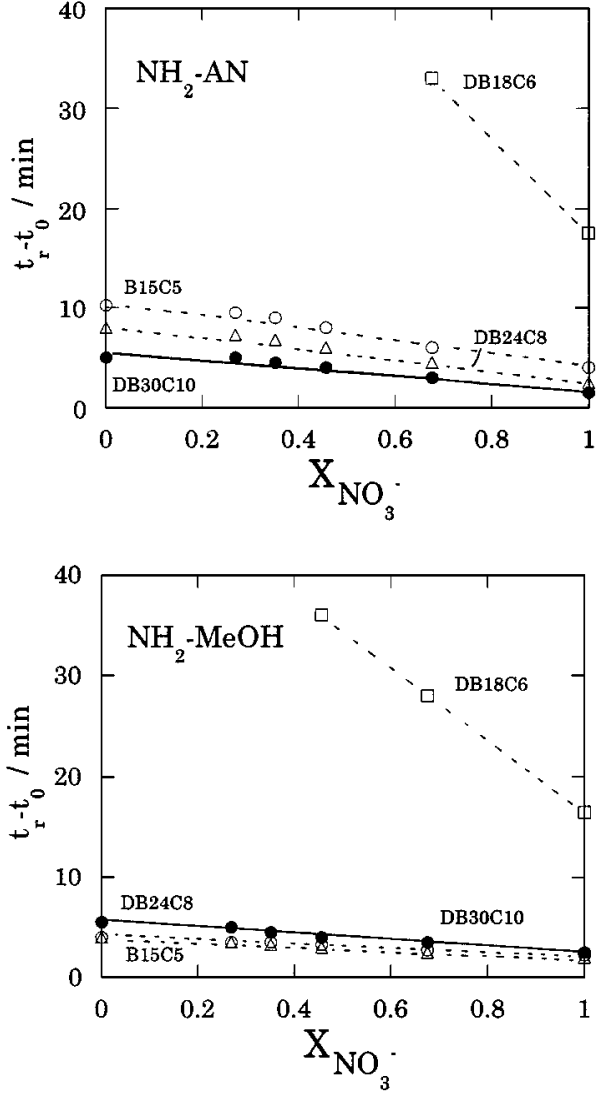

Fig. 5 Dependence of the retention of crown ethers on the molar ratio of $\mathrm{NO}_{3}{ }^{-}$in the $-\mathrm{NH}_{3}{ }^{+}$resin with mixed counteranions $\left(\mathrm{NO}_{3}^{-}\right.$and $\left.\mathrm{ClO}_{4}^{-}\right)$. The upper, in AN. The lower, in $\mathrm{MeOH}$.

and (3), the contribution from these to the overall retention of crown ethers is represented by $K_{3} / K_{1}$. We previously reported that the $-\mathrm{NH}_{3}{ }^{+}$sites behave like tetraalkylammonium ion-type anion-exchange sites after crown ether complexation..$^{28}$ It is known that the interaction of smaller anions with tetraalkylammonium ions is stronger than that of larger anions in AN. ${ }^{29}$ In contrast, the interaction of smaller anions with tetraalkylammonium ions is weaker in hydrogenbonding solvents, such as $\mathrm{MeOH} .{ }^{29}$ Thus, unfavorable effects of $\mathrm{NO}_{3}{ }^{-}$counteranions on the crown ether retention in AN are mostly compensated by the stabilization by equilibrium (3). This contribution from equilibrium (3) is not very large in $\mathrm{MeOH}$, where equilibrium (1) is also less important.

The retention of crown ethers was studied with mixed-counteranion $\left(\mathrm{NO}_{3}{ }^{-}\right.$and $\left.\mathrm{ClO}_{4}{ }^{-}\right)$resins. Figures 5 -7 show the dependence of crown ether retention with $-\mathrm{NH}_{3}{ }^{+}$, $-\mathrm{en}^{2+}$, and $-\mathrm{dien}^{3+}$ resins on the molar ratio of $\mathrm{NO}_{3}{ }^{-}$in the stationary phases $\left(X_{\mathrm{NO} 3(\mathrm{r})}\right)$. Plots for B15C5, DB18C6, and DB24C8 seem linear in all cases. The linear relations between capacity factors $(k)$ and $X_{\mathrm{NO} 3(\mathrm{r})}$ can simply be explained by the relation

$$
k=X_{\mathrm{A}} k_{\mathrm{A}}+X_{\mathrm{B}} k_{\mathrm{B}},
$$



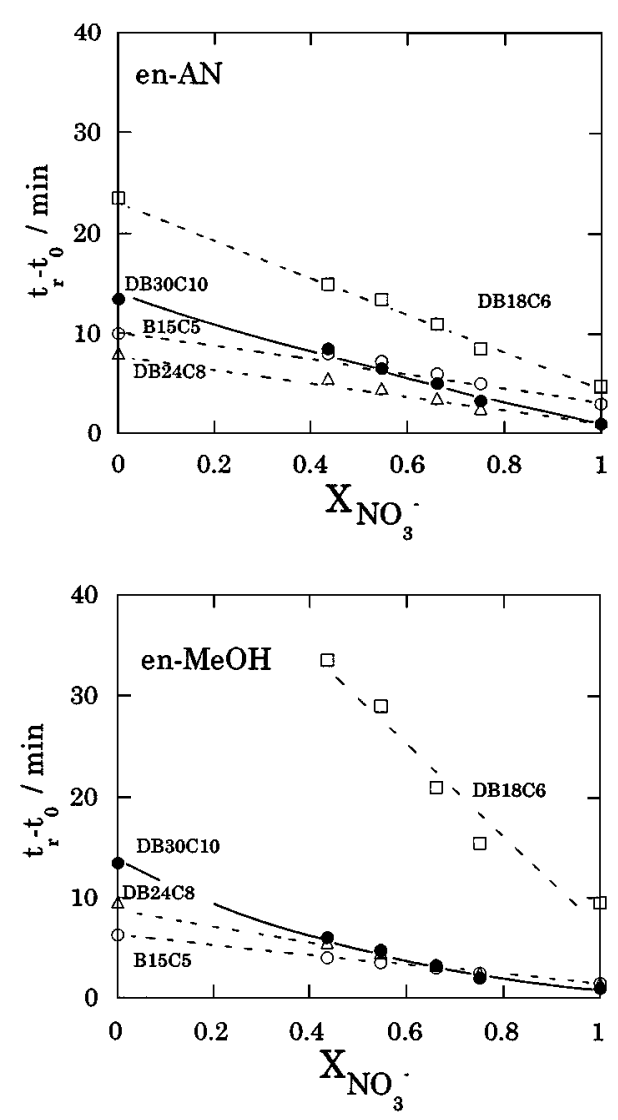

Fig. 6 Dependence of the retention of crown ethers on the molar ratio of $\mathrm{NO}_{3}^{-}$in the $-\mathrm{en}^{2+}$ resin with mixed counteranions $\left(\mathrm{NO}_{3}^{-}\right.$and $\left.\mathrm{ClO}_{4}^{-}\right)$. The upper, in AN. The lower, in $\mathrm{MeOH}$.

where $X$ is the molar ratio of a counteranion in a resin, and subscripts A and B refer to the counteranions. This linear relation may suggest that the similar mechanisms govern the overall retention of crown ethers, even in different solvents and with different counteranions.

In contrast, the retention of DB30C10 on the $-\mathrm{en}^{2+}$ resin is not explained by Eq.(4). DB30C10 is much more strongly retained on the $\mathrm{ClO}_{4}^{-}$resins than expected by extrapolation from the retention on $\mathrm{NO}_{3}{ }^{-}$resins. As stated above, the simultaneous accommodation of two ammonium groups in an $-\mathrm{en}^{2+}$ molecule by DB30C10 is an important feature in this particular case. This specific interaction is seen only with $\mathrm{ClO}_{4}{ }^{-}$counteranions, as shown in Table 1. If the distribution of counteranions in the resin is homogeneous, the possibility that a given $-\mathrm{en}^{2+}$ molecule has two $\mathrm{ClO}_{4}^{-}$as the counteranions is given by $\left(X_{\mathrm{ClO} 4}\right)^{2}$. The regression analysis for the curves of DB30C10 illustrated in Fig. 6 gives the following relations:

$$
\begin{array}{ll}
t=10.3\left(X_{\mathrm{ClO} 4}\right)^{2}+7.61 X_{\mathrm{ClO} 4}+0.965 & \text { in } \mathrm{AN} \\
t=8.48\left(X_{\mathrm{ClO} 4}\right)^{2}+4.22 X_{\mathrm{ClO} 4}+0.848 & \text { in } \mathrm{MeOH},
\end{array}
$$

where $t$ denotes the corrected retention times, taken as the $y$-coordinate in Fig. 6 . Thus, the retention of
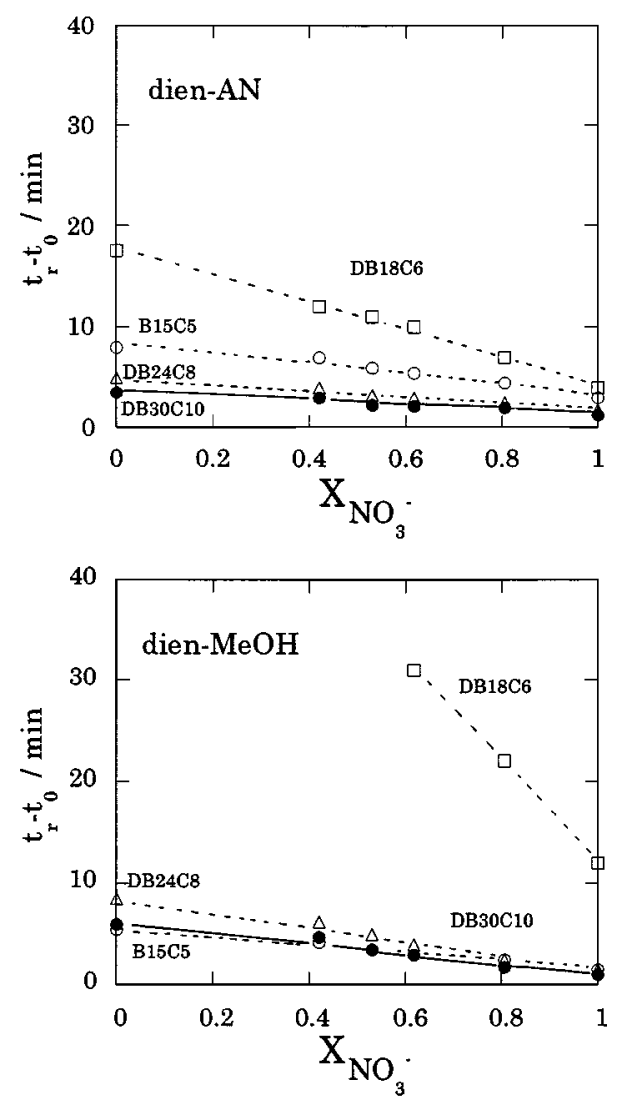

Fig. 7 Dependence of the retention of crown ethers on the molar ratio of $\mathrm{NO}_{3}{ }^{-}$in the - dien $^{3+}$ resin with mixed counteranions $\left(\mathrm{NO}_{3}^{-}\right.$and $\left.\mathrm{ClO}_{4}^{-}\right)$. The upper, in AN. The lower, in $\mathrm{MeOH}$.

DB30C10 on the $-\mathrm{en}^{2+}$ resin should be expressed by a quadratic equation of $X_{\mathrm{ClO} 4}$ rather than a linear equation such as Eq.(4). The above equations indicate that the contribution from the diammonium-DB30C10 complex formation to its retention is comparable to that from the monoammonium-DB30C10 complex formation.

In conclusion, the retention of crown ethers on polyammonium ion resins has been discussed from viewpoints of ion-pair and complex formations. The ion-pair formation of the active sites with counteranions basically competes with the complexation of crown ethers. However, after complexation, counteranions again form ion-pairs with active sites complexed by crown ethers, and stabilizes the overall retention of the crown ethers. These two contributions happen to be almost canceled out; the counteranion effects are much smaller than expected from ion-pair formation. The retention selectivity thus reflects the complexation selectivity in most cases. The present chromatographic method can therefore be used for a rough estimation of the complexation ability of polyethers and for the screening of useful polyethers having specific selectivity toward particular target molecules, if the target cations can be chemically anchored on the chromatographic stationary phases. 
This work has been supported in part by a Grant-in-Aid for the Scientific Researches from the Ministry of Education, Science, Culture, and Sports, Japan. We thank the Japan Society for the Promotion of Science for the fellowship to SSB.

\section{References}

1. C. J. Pedersen, J. Am. Chem. Soc., 89, 7017 (1967).

2. K. Ozutsumi and S. Ishiguro, Bull. Chem. Soc. Jpn., 65, 1173 (1992)

3. B. L. Haymore, J. D. Lamb, R. M. Izatt, J. J. Christensen, Inorg. Chem., 21, 1598 (1982).

4. K. Ohtsu, T. Kawashima and K. Ozutsumi, J. Chem. Soc. Faraday Trans., 91, 4375 (1995).

5. K. M. Doxsee, H. R. Wierman and T. J. R. Weakley, J. Am. Chem. Soc., 114, 5165 (1992).

6. Y. Miyazaki and H. Matsuura, Bull. Chem. Soc. Jpn., 64 , 288 (1991)

7. R. D. Rogers, A. H. Bond, S. Aguinaga and A. Reyes, $J$. Am. Chem. Soc., 114, 2967 (1992).

8. L. J. Rodringuez, E. M. Eyring and S. Petrucci, J. Phys Chem., 93, 5916 (1989).

9. L. X. Dang and P. A. Kollman, J. Phys. Chem., 99, 55 (1995).

10 L. Troxler and G. Wipff, J. Am. Chem. Soc., 116, 1468 (1994).

11. R. M. Izatt, S. J. Bradshaw, S. A. Nielsen, J. D. Lamb and J. J. Christensen, Chem. Rev., 85, 271 (1985) and references therein.

12. S. Tsurubou, M. Mizutani, Y. Kadota, T. Yamamoto, S Umetani, T. Sasaki, Q. T. H. Le and M. Matsui, Anal.
Chem., 67, 1465 (1995).

13. M. M. Wienk, T. B. Stolwijk, E. J. R. Sudhölter and D. N. Reinhoudt, J. Am. Chem. Soc., 112, 797 (1990).

14. M. Billah, T. Honjo and K. Terada, Fresenius' J. Anal. Chem., 347, 107 (1993).

15. A. Ohki, J.-P. Lu and R. A. Bartsch, Anal. Chem., 66, 651 (1994).

16. K. Suzuki, K. Sato, H. Hisamoto, D. Siswanta, K. Hayashi, N. Kasahara, K. Watanabe, N. Yamamoto and H. Sasakura, Anal. Chem., 68, 208 (1996).

17. M. A. Arnold, Anal. Chem., 64, 1015A (1992) and references therein.

18. T. Hayashita, Bunseki, 276 (1995).

19. T. Okada, Anal. Chem., 62, 327 (1990).

20. T. Okada and T. Usui, Anal. Chem., 66, 1654 (1994).

21. T. Okada, Macromolecules, 23, 4216 (1990).

22. T. Okada, J. Phys. Chem. B, 102, 3053 (1998).

23. T. Okada and T. Usui, J. Chem. Soc. Faraday Trans., 92 4977 (1996).

24. T. Okada, J. Phys. Chem. B, 101, 7814 (1997).

25. T. Okada, J. Chromatogr. A, 695, 309 (1995).

26. M. S. Islam, R. A. Pethrick, D. Pugh and M. J. Wilson, $J$. Chem. Soc. Faraday Trans., 94, 39 (1998).

27. T. Kowall and A. Geiger, J. Phys. Chem., 99, 5240 (1995).

28. T. Okada, J. Chromatogr. A, 758, 29 (1997).

29. S. Okazaki and I. Sakamoto, "Yobai to Ion (Solvents and Ions, in Japanese)"; Taniguchi Insatsu: Matsue, 1990, and references therein.

(Received December 24, 1998) (Accepted March 4, 1999) 